\title{
ARTICLE
}

\section{Dataset of TLD badge response and hair activation for criticality accident neutron dosimetry}

\author{
Norio Tsujimura $^{\mathrm{a}^{*}}$, Fumiaki Takahashi ${ }^{\mathrm{b}}$ and Chie Takada ${ }^{\mathrm{a}}$ \\ a Japan Atomic Energy Agency, 4-33 Muramatsu, Tokai-mura, Naka-gun, Ibaraki-ken, 319-1194, Japan; 'bapan Atomic Energy \\ Agency, 2-4 Shirakata, Tokai-mura, Naka-gun, Ibaraki-ken, 319-1195, Japan
}

\begin{abstract}
Criticality accident neutron dosimetry is based on measurement conducted using personal dosemeters and biological samples of persons exposed to neutrons from an accidental criticality. The authors computed the response functions of a personal dosemeter (NCL-TLD badge) and hair (sulfur) activation per unit incident neutron fluence, and established a response dataset compiled using 140 neutron spectra that are likely to be encountered in a criticality accident.
\end{abstract}

Keywords: criticality accident; neutron dosimetry; MCNP; TLD; indium-115m; phosphorus-32

\section{Introduction}

The Nuclear Fuel Cycle Engineering Laboratories (NCL), Japan Atomic Energy Agency, has never experienced a criticality accident. However, criticality dosimetry procedures have been developed as a precaution. The first step to be taken immediately after an accident is screening of severely exposed persons by direct survey of the indium foil incorporated into their personal dosemeters. The screening procedure is followed by normal processing of the personal dosemeter and, if necessary, in-depth dosimetry procedures, such as measurements of ${ }^{115 \mathrm{~m}} \mathrm{In}$ in the indium foil, ${ }^{24} \mathrm{Na}$ in the blood/whole-body, and ${ }^{32} \mathrm{P}$ in hair [1]. The sole use of either of these dosimetry techniques is generally unsatisfactory because any one of these does not fully cover the entire energy range of interest. Therefore, to achieve the best dose estimates, it is necessary to use all of these techniques with assumptions on the neutron spectrum that provides good internal consistency among the dose results evaluated independently using each of these techniques.

This paper describes an explanation of the neutron detection techniques used in NCL, a method of computation of their response functions, and the computational results of spectrum-folded responses for criticality accident neutron dosimetry.

\section{Materials and methods}

\subsection{Personal dosemeter and biological samples}

The criticality accident dosimetry procedures at NCL consist of quick screening of potentially exposed

*Corresponding author. Email: tsujimura.norio@jaea.go.jp persons; normal processing of personal dosemeters to provide early but preliminary dose estimates; and measurements of ${ }^{115 \mathrm{~m}} \mathrm{In}$ in indium foil, ${ }^{24} \mathrm{Na}$ in the blood/whole-body, and ${ }^{32} \mathrm{P}$ in hair to make final dose estimates. This order corresponds roughly to the degree of rapidness of measurements and the short order of half-lives of the activation products.

The personal dosemeters used in NCL are the TLD badge, constituted of two Panasonic thermoluminescence dosemeters, namely, UD-808P for beta/gamma rays and UD-809P for neutrons, on which primary reliance is placed in the early stage of criticality dosimetry. Gamma dose is evaluated using the reading of the ${ }^{7} \mathrm{Li}_{2}{ }^{11} \mathrm{~B}_{4} \mathrm{O}_{7}: \mathrm{Cu}$ phosphor covered with a $1 \mathrm{~g} / \mathrm{cm}^{2}$ resin filter, and neutron dose is evaluated from the net reading of the ${ }^{6} \mathrm{Li}_{2}{ }^{10} \mathrm{~B}_{4} \mathrm{O}_{7}$ : $\mathrm{Cu}$ phosphor surrounded by a neutron shield (cadmium), with the exception of the neutron-transparent window (tin) behind the phosphor. The units used for criticality dosimetry are Gy, not Sv for routine dosimetry. The TLD badge also contains a small piece of indium foil for dual purposes: screening of exposed persons and fast neutron dosimetry by using the reactions of ${ }^{115} \operatorname{In}(n, \gamma)^{116 \mathrm{~m}} \mathrm{In}$ and ${ }^{115} \operatorname{In}\left(n, n^{\prime}\right)^{115 \mathrm{~m}} \mathrm{In}$, respectively. The latter is the reaction of interest in this paper, that is, threshold reaction with fast neutrons having energies $>\sim 1 \mathrm{MeV}$, and the resulting ${ }^{115 \mathrm{~m} I n}$ with a half-life of $\sim 4.5 \mathrm{~h}$ may be measured by high-resolution gamma spectrometry.

Moreover, analysis of ${ }^{24} \mathrm{Na}$ (a half-life: $\sim 15 \mathrm{~h}$ ) in blood/whole-body, produced via the ${ }^{23} \mathrm{Na}(n, \gamma)^{24} \mathrm{Na}$ reaction, is performed based on the priority determined according to the preliminary dose estimated using the TLD badge. In addition, supplemental hair activation analysis of ${ }^{32} \mathrm{P}$ with a half-life of $\sim 14 \mathrm{~d}$, produced via the ${ }^{32} \mathrm{~S}(n, p){ }^{32} \mathrm{P}$ reaction with a threshold energy of $\sim 2.0$ $\mathrm{MeV}$, may be performed at the later dosimetry stage. 


\subsection{Calculation of response functions}

To evaluate the response functions of the TLD badge and hair activation as a function of neutron energy and angle of incidence, two simulation models are created using the MCNP (Monte Carlo N-Particle) code.

The first model, revised as an extension of previous model [1], is constructed of a single TLD badge (including Panasonic UD-808 and UD-809 dosemeters housed in a Panasonic UD-887 plastic case and a piece of indium foil ( $\sim 0.36 \mathrm{~g})$ inserted into the internal slot of the case) placed on the center of the front surface of a water-filled $30 \times 30 \times 15 \mathrm{~cm}^{3}$ phantom. The UD-809 neutron dosemeter model includes $\mathrm{Li}_{2} \mathrm{~B}_{4} \mathrm{O}_{7}: \mathrm{Cu}$ phosphors, an ABS resin holder, and cadmium and tin filters; they are based accurately on the actual dimensions, with the exception of the phosphors in which the phosphor volumes are enlarged with their actual masses preserved. The number of ${ }^{6} \mathrm{Li}(n, \alpha)$ and ${ }^{10} \mathrm{~B}(n, \alpha)$ reactions in the phosphor of interest, which exclusively measures albedo neutrons, is scored by the track length estimate tally (F4) with the multiplier of the microscopic cross-sections and the atomic densities of ${ }^{6} \mathrm{Li}$ and ${ }^{10} \mathrm{~B}$. The conversion factor from the number of the $(n, \alpha)$ reactions in the phosphor to the reading in units of $\mathrm{Sv}{ }^{137} \mathrm{Cs} \gamma$-equivalent, denoted hereinafter as $\mathrm{Sv} \gamma$-eq., is determined by comparing the experimental results obtained in a thermal neutron field with the computational results obtained using the same irradiation setup. The numbers of ${ }^{115} \operatorname{In}(n, \gamma)^{116 \mathrm{~m}} \mathrm{In}$ and ${ }^{115} \operatorname{In}\left(n, n^{\prime}\right)^{115 \mathrm{~m}} \mathrm{In}$ reactions in the indium foil are also tallied by the F4 tally with the multipliers of the microscopic cross-sections and the atomic density of ${ }^{115} \mathrm{In}$, and then converted to the activities of ${ }^{116 \mathrm{~m}} \mathrm{In}$ and $115 \mathrm{~m}$ In by multiplying their respective decay constants.

The second model consists of small cells placed on the front, side, and back of the head phantom [2]. One gram of human hair contains $43.6 \mathrm{mg}$ of sulfur and $0.171 \mathrm{mg}$ of phosphorus [3]; therefore, the competitive reactions of ${ }^{31} \mathrm{P}(n, \gamma)^{32} \mathrm{P}$ and $\left.{ }^{31} \mathrm{P}(n, p)\right)^{31} \mathrm{Si}$ are considered in addition to the ${ }^{32} \mathrm{~S}(n, p){ }^{32} \mathrm{P}$ reaction of interest. The activities of ${ }^{32} \mathrm{P}$ and ${ }^{31} \mathrm{Si}$ per gram of hair are also calculated in a manner similar to that of activated indium.

Neutrons with selected energy intervals ranging from thermal to $15 \mathrm{MeV}$ are generated as a parallel beam source covering the entire computation models. Moreover, the models are rotated horizontally for angular response calculations in increments of $30^{\circ}$. The nuclear data library used is JENDL-3.2 [4] and the dosimetry data is JENDL/D-99 [5].

\subsection{Spectrum-folding of response functions}

The response functions computed are spectrum-folded for most likely 140 neutron spectra, cited from the neutron spectrum database of the program RADAPAS (RApid Dose Assessment Program from Activated Sodium in Criticality Accidents) [6]. The neutron absorbed dose corresponding to the selected neutron spectrum is also calculated using the neutron fluence to the absorbed dose conversion factor (Table XIV, column 4, of IAEA's manual [7]).

\section{Results and discussion}

\subsection{Response functions}

Figure 1 shows the response functions per unit neutron fluence of (a) TLD, (b) ${ }^{115 \mathrm{~m}} \mathrm{In}$ and ${ }^{116 \mathrm{~m}} \mathrm{In}$ in the TLD badge, and (c) ${ }^{32} \mathrm{P}$ and ${ }^{31} \mathrm{Si}$ in $1 \mathrm{~g}$ of human hair (collected from the forehead) for the incident angles of $0^{\circ}$ (AP), $+90^{\circ}$ (RLAT), $+270^{\circ}$ or $-90^{\circ}$ (LLAT), and $+180^{\circ}$ (PA). In this figure, the values of the neutron-induced activities are those immediately after instantaneous neutron irradiation.
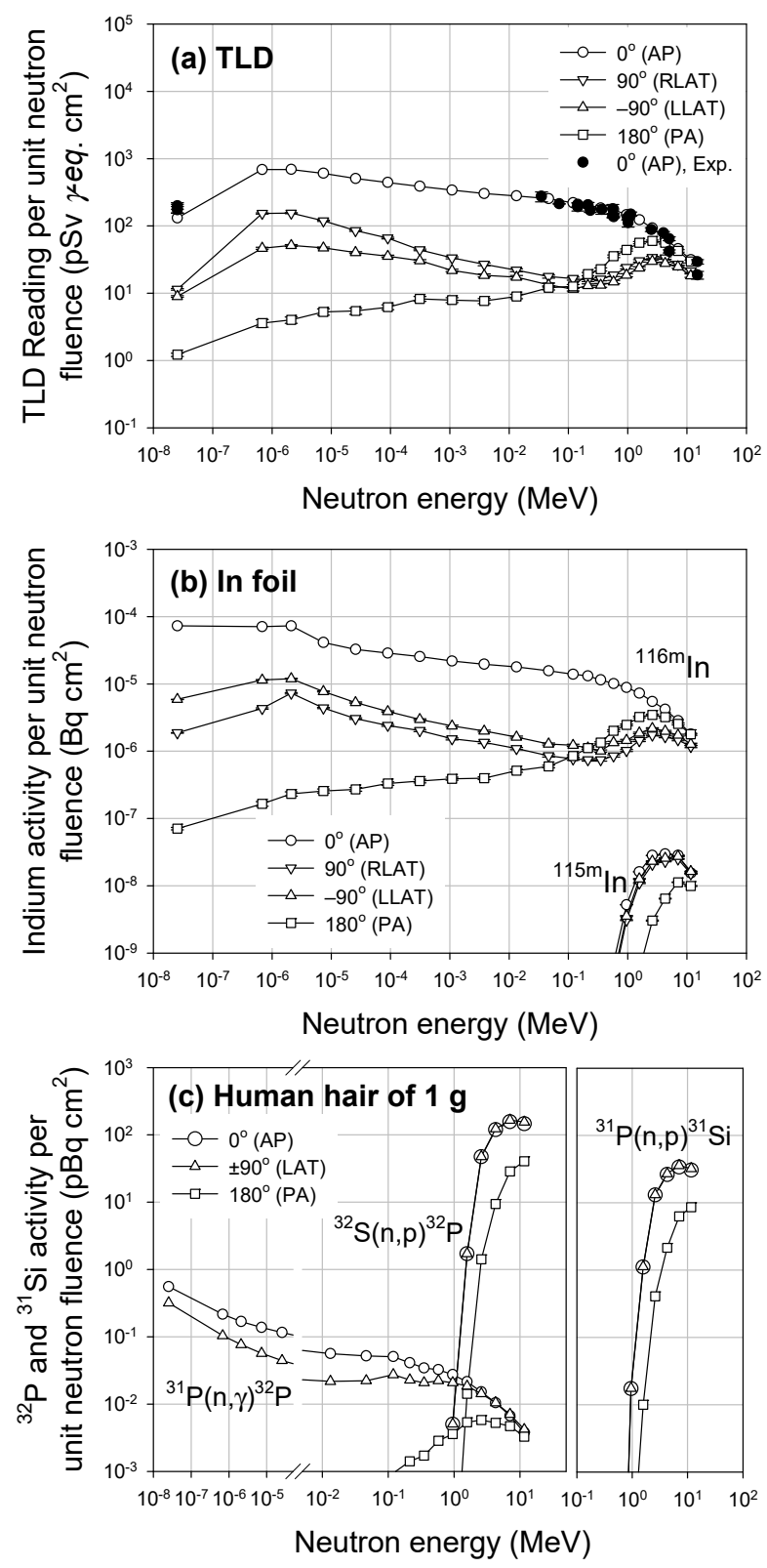

Figure 1. Response functions per unit neutron fluence of (a) TLD, (b) ${ }^{115 \mathrm{~m} I n}$ and ${ }^{116 \mathrm{~m}} \mathrm{In}$ in the TLD badge, and (c) ${ }^{32} \mathrm{P}$ and ${ }^{31} \mathrm{Si}$ in $1 \mathrm{~g}$ of human hair. 
As shown in Figure 1(a) along with the experimental responses obtained using accelerator-produced monoenergetic and graphite-pile-moderated thermal neutrons, the TLD exhibits relatively flat responses over the slow-to-intermediate energy region, decreasing gradually with increasing neutron energy. Its responses to lateral and back irradiation are less sensitive. Minor differences in the TLD responses between both lateral irradiations (RLAT and LLAT) are ascribed to the asymmetry of the badge case construction.

There are many similarities between the characteristics of ${ }^{115 \mathrm{~m}} \mathrm{In}$ and those of ${ }^{32} \mathrm{P}$, as shown in Figures 1(b) and (c); above the threshold neutron energies of a few $\mathrm{MeV}$, both the responses increase rapidly with energies up to $\sim 10 \mathrm{MeV}$ and there are no marked differences between the frontal and the lateral irradiations, with attenuated responses to the back irradiation. As for the competitive reaction products, the interferences of ${ }^{116 \mathrm{~m}} \mathrm{In}$ with a half-life of $54 \mathrm{~min}$ and ${ }^{31} \mathrm{Si}$ with a half-life of $2.6 \mathrm{~h}$ may be mitigated by delaying the counting of ${ }^{115 \mathrm{~m}} \mathrm{In}$ and ${ }^{32} \mathrm{P}$, respectively, for a few hours. The influence of ${ }^{32} \mathrm{P}$ originating from ${ }^{31} \mathrm{P}$ may be virtually negligible, except for the highly thermalized neutron fields, because the amount of ${ }^{32} \mathrm{P}$ originating from ${ }^{32} \mathrm{~S}$ is usually predominant.

\subsection{Spectrum-folded responses}

A total of 140 neutron spectra used for folding calculations taken from the program RADAPAS were code-numbered as \#1-8, the source neutrons that leak from a postulated criticality assembly (metallic uranium with a radius of $8.74 \mathrm{~cm}$ and aqueous uranium with radii of 17.4-50.0 cm); \#9-48, those transmitted through ordinary concrete $(10-50 \mathrm{~cm}$ thickness); \#49-80, through heavy concrete (10-50 cm thickness); \#81-114, through steel (5-50 cm thickness); and \#115-140, through lead $(5-50 \mathrm{~cm}$ thickness) [6]. Figure 2 summarizes the neutron-spectral features of the 140 spectra, expressed with the fractional fluence (\%) within the given energy intervals and the average neutron energy as the ordinates and the spectrum number as the abscissa. With hydrogenous shielding such as concrete, the average neutron energy shifts generally toward lower energies owing to the increased number of thermal-to-intermediate energy neutrons with increasing amounts of shielding; but for a thickness $>30 \mathrm{~cm}$, the average energy remains unchanged because there is little change in the peak spectrum around $2 \mathrm{MeV}$. With steel and lead shielding, the fast neutron component is reduced drastically by inelastic scattering with increasing shielding thickness, and the neutrons transmitted through both materials show closely comparable spectra in shape, except for the existence of small amounts of thermal neutrons.

Figure 3 shows the spectrum-folded responses per unit neutron absorbed dose of (a) TLD, (b) ${ }^{115 \mathrm{~m}} \mathrm{In}$ in the TLD badge, and (c) ${ }^{32} \mathrm{P}$ in $1 \mathrm{~g}$ of human hair (collected from the forehead) for the 140 neutron spectra. The values of the activities are those for instantaneous neutron irradiation.

For hydrogen-moderated neutron spectra (\#2-80), the TLD responses to AP irradiation are relatively less dependent on the assembly radius or the shielding conditions, varying from a low value of $10 \mathrm{~Sv} \gamma$-eq./Gy for spectrum \#2 to a high value of $22 \mathrm{~Sv} \gamma$-eq./Gy for spectrum \#73. For steel/lead shielding, information on the shielding thickness is required to account for the large spectral variations expected in the response. Indium- $115 \mathrm{~m}$ and ${ }^{32} \mathrm{P}$ have the advantage of a considerably more constant response, for example, $440-510 \mathrm{~Bq} / \mathrm{Gy}$ for ${ }^{115 \mathrm{~m}} \mathrm{In}$ and $0.86-1.2 \mathrm{~Bq} / \mathrm{Gy}$ for ${ }^{32} \mathrm{P}$, to AP irradiation, as in spectra \#1-80. However, for thick steel or lead shielding, these responses drop to nearly zero, which is of little value for neutron dosimetry.

To arrive at the final dose estimates, the person's orientation with regard to the criticality assembly at the time of the accident must be known for selecting appropriate responses. That may be deduced by comparing the measurements of different angular

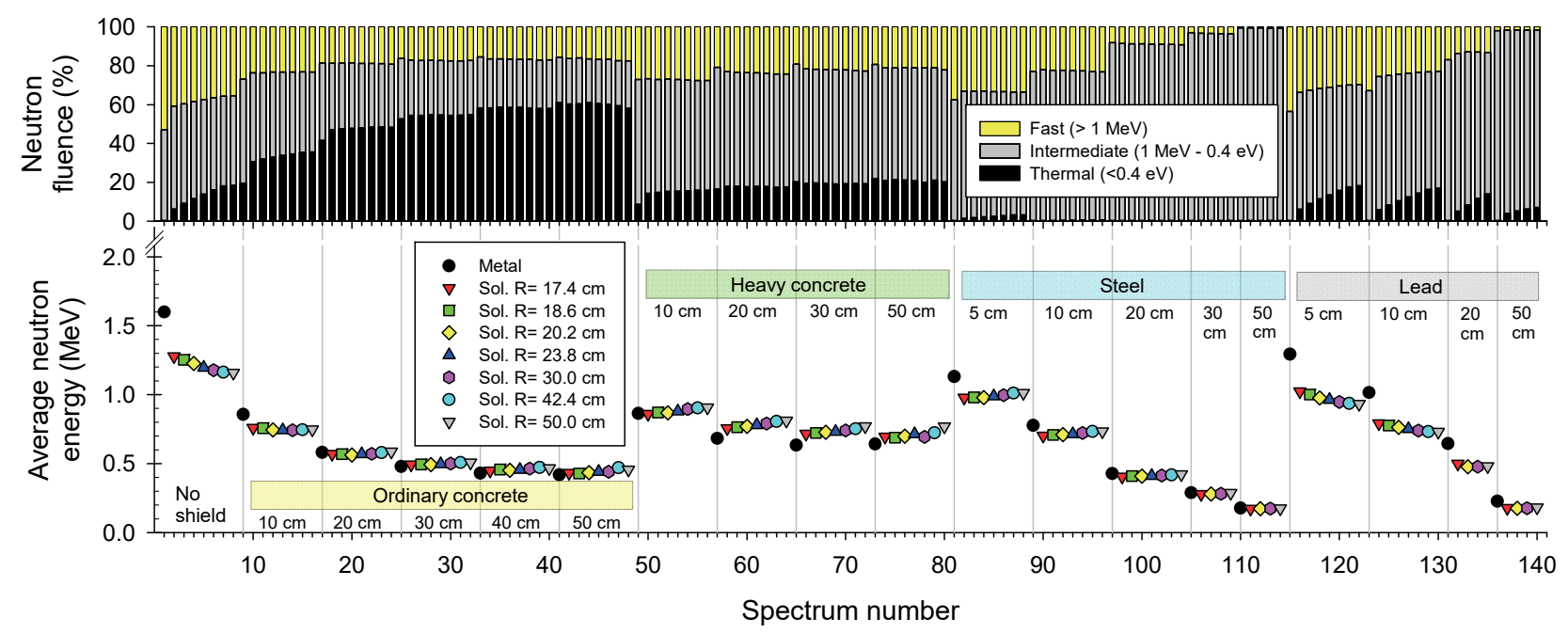

Figure 2. Neutron-spectral features of 140 spectra: (upper) fractional fluence (\%) within given energy intervals and (lower) average neutron energy. 

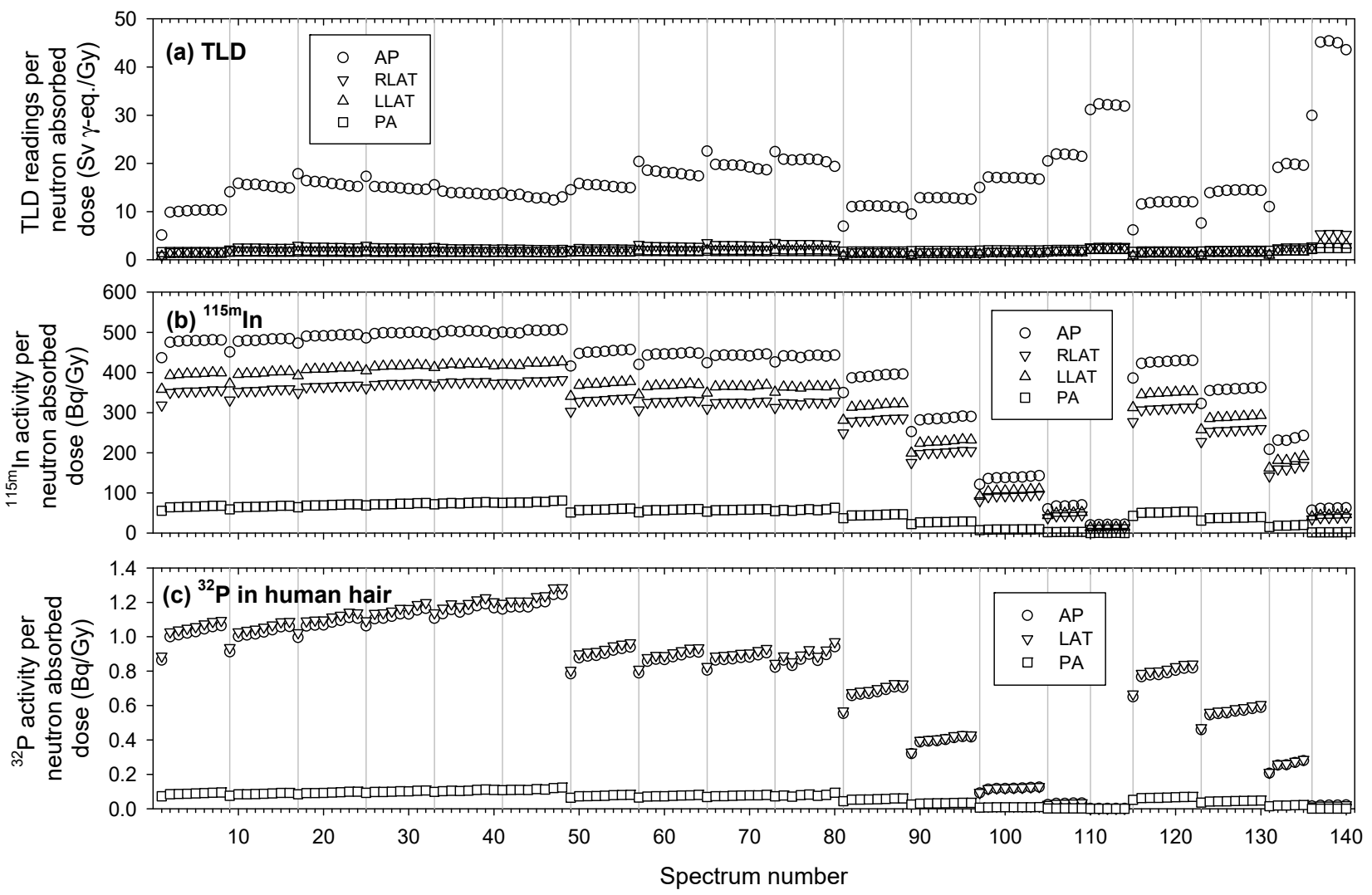

Figure 3. Spectrum-folded responses per unit neutron absorbed dose of (a) TLD, (b) ${ }^{115 \mathrm{~m} I n}$ in the TLD badge, and (c) ${ }^{32} \mathrm{P}$ in $1 \mathrm{~g}$ of human hair (collected from the forehead) for 140 neutron spectra.

response characteristics: the TLD of poor angular response at angles other than $\mathrm{AP},{ }^{115 \mathrm{~m} I n}$ of reduced response to $\mathrm{PA}$, and ${ }^{24} \mathrm{Na}$ of relatively orientation-independent response [6]. This assumed orientation can also be confirmed by analyzing ${ }^{32} \mathrm{P}$ in hair samples taken at various positions.

\section{Conclusion}

The authors computed the neutron response functions of the TLD badge, ${ }^{115 \mathrm{~m}}$ In activity in the TLD badge, and ${ }^{32} \mathrm{P}$ activity in $1 \mathrm{~g}$ of human hair for the purpose of neutron dosimetry in a criticality accident. These response datasets compiled under the same neutron-spectral conditions as those in the program RADAPAS would facilitate direct comparisons among the responses of different neutron detection techniques in conjunction with the ${ }^{24} \mathrm{Na}$ response data of RADAPAS. If the dose results evaluated independently from each of these techniques provide good internal consistency when using a neutron spectrum selected from the RADAPAS database, the selected spectrum and the relevant spectrum-folded responses are judged to be good choices. If not, the selection process should be repeated to improve the consistency.

\section{Acknowledgements}

This work was supported by a Labor Accident
Disease and Clinical Research Grant.

\section{References}

[1] C. Takada, N. Tsujimura and S. Mikmai, Recalibration of indium foil for personnel screening in criticality accidents, Radiat. Prot. Dosim. 144 (2011), pp. 575-579.

[2] A.L. Reed, Medical physics calculations with MCNP: A primer, LA-UR-07-4133, Los Alamos National Laboratory, (2007).

[3] T. Maruyama, Dose evaluation for external exposure in radiation accidents, NIRS-M-71, National Institute of Radiological Science, (1989).

[4] Nakagawa, T. et al., Japanese evaluated nuclear data library version 3 revision 2. J. Nucl. Sci. Technol. 32 (1995), pp. 259-1271.

[5] K. Kobayashi, et al., JENDL dosimetry file 99 (JENDL/D-99), JAERI 1344, Japan Atomic Energy Research Institute, (2002).

[6] F. Takahashi, Program for Rapid Dose Assessment in Criticality Accident, RADAPAS, JAEA-Data/Code 2006-019, Japan Atomic Energy Agency, (2006).

[7] IAEA, Dosimetry for Criticality Accidents-A Manual, Technical Report Series No. 211, International Atomic Energy Agency, (1982). 